University of Warwick institutional repository

This paper is made available online in accordance with

publisher policies. Please scroll down to view the document

itself. Please refer to the repository record for this item and our

policy information available from the repository home page for further information.

To see the final version of this paper please visit the publisher's website. Access to the published version may require a subscription.

Author(s): Ngianga-Bakwin Kandala, Ludwig Fahrmeir, Stephan

Klasen, Jan Priebe

Article Title: Geo-additive models of childhood undernutrition in three sub-Saharan African countries

Year of publication: 2008

Link to published version: http://dx.doi.org/10.1002/psp.524

Publisher statement: The definitive version is available at

www3.interscience.wiley.com 


\title{
Geo-additive models of Childhood Undernutrition in three Sub-Saharan African Countries
}

\author{
Ngianga-Bakwin Kandala ${ }^{1}$, Ludwig Fahrmeir, ${ }^{2}$ Stephan Klasen ${ }^{3}$ and Jan Priebe ${ }^{3}$ \\ ${ }^{1}$ Clinical Sciences Research Institute, Warwick Medical School, University of Warwick, CV2 2DX, Coventry, UK. \\ ${ }^{2}$ Department of Statistics, University of Munich, Germany. \\ ${ }^{3}$ Department of Economics, University of Göttingen, Germany.
}

\begin{abstract}
We investigate the geographical and socioeconomic determinants of childhood undernutrition in Malawi, Tanzania and Zambia, three neighbouring countries in Southern Africa using the 1992 Demographic and Health Surveys. In particular, we estimate models of undernutrition jointly for the three countries to explore regional patterns of undernutrition that transcend boundaries, while allowing for country-specific interactions. We use geo-additive regression models to flexibly model the effects of selected socioeconomic covariates and spatial effects. Inference is fully Bayesian based on recent Markov chain Monte Carlo techniques.

While the socioeconomic determinants generally confirm findings from the literature we find distinct residual spatial patterns that are not explained by the socioeconomic determinants. In particular, there appears to be a belt transcending boundaries and running from Southern Tanzania to North-eastern Zambia which exhibits much worse undernutrition. These findings have important implications for planning as well as in the search for left-out variables that might account for these residual spatial patterns.
\end{abstract}

Keywords: Sub-Saharan Africa; Geo-additive models; undernutrition; spatial statistics; semi-parametric Bayesian analysis. 


\section{INTRODUCTION}

Malawi, Tanzania and Zambia are neighbouring low-income countries in Southern Africa, all belonging to the poorest countries in the world, with very poor education, health, and human development indicators. They have been affected by years of economic stagnation and decline with negative per capita income growth rates throughout the 1980s and early 1990s, and have also experienced deterioration in health and education indicators (World Bank, 2000). ${ }^{1}$ Chronic undernutrition is a serious problem in all three countries, affecting some $48.7 \%$ of children in Malawi, $46.7 \%$ in Tanzania, and 39.6\% in Zambia. Given the centrality of undernutrition for child well-being, it is critical to understand its determinants.

When modelling the determinants of undernutrition, one can distinguish between immediate, intermediate, and underlying determinants (see UNICEF, 1998). While undernutrition is always immediately related to either insufficient nutrient intake or the inability of the body to absorb nutrients (primarily due to illness), these are themselves caused by problems related to food security, care practices, and the health environment at the household level, which themselves are influenced by the socioeconomic and demographic situation of households and communities (UNICEF; 1998; Smith and Haddad, 1999, Klasen, 1999). In order to capture this complex chain of causation, researchers have either focused on a particular level of causality (e.g. Smith and Haddad, 1999; Moradi, 1999, Pelletier, 1999), have estimated structural equations that address interactions between the different levels (e.g. Guilkey and Riphahn, 1998), have used graphical chain models to assess the causal pathways (Caputo, et al. 2003), or have used

\footnotetext{
${ }^{1}$ More recently, they have also been severely affected by the AIDS pandemic.
} 
multi-level modelling techniques (e.g. Nyovani et al. 1999, Harttgen and Misselhorn, 2006).

In this paper, we estimated a model that mainly focuses on factors that are underlying determinants of undernutrition. There is also some discussion in the literature on non-linear effects of some of these covariates although they are rarely investigated thoroughly (e.g. Smith et al. 2001; WHO, 1995, Stephenson, 1999).

The particular innovations of the paper are threefold: first, through the use of our empirical methods we are able to investigate non-linear effects more flexibly than most previous work. Second, the methods also allow us to investigate the spatial pattern of undernutrition, prior to and after controlling for the socioeconomic covariates. This enables us to determine to what extent the substantial spatial pattern of undernutrition is driven by the socioeconomic factors we were considering. Third, by using data from three adjacent countries in the same year, we are able to investigate the relative importance of country-specific socio-economic factors and government policies vis-à-vis geographical factors that transcend boundaries.

\section{DATA, MEASUREMENT OF UNDERNUTRITION, AND COVARIATES}

The data we use are from the 1992 round of the Demographic and Health Surveys (DHS) undertaken in the three Sub-Saharan African countries of Malawi, Tanzania and Zambia. The DHSs collect information on a nationally representative sample of women in childbearing age (15-49). The questionnaire collects socioeconomic indicators for the respondent and her partner as well as the household she resides in and then gathers a large amount of information on fertility patterns, health and care practices, health knowledge, and assesses the anthropometric status of all children of these women who 
were born within the past five years. Data collection and analysis is highly standardized and supported from Macro, Inc. on behalf of the US Agency for International Development. The DHS have long been considered to be the most reliable sources of demographic, nutrition, and child health and mortality information in developing countries, particularly in Africa where administrative data on these issues are largely incomplete or missing. Unfortunately, the surveys do not generate an income variable and we therefore rely on a household asset index as a proxy for the income situation of the households which has been found to be quite reliable by Filmer and Pritchett (2001). The 1992 DHS data sets of Malawi, Tanzania and Zambia are pooled to form one data set with the same socio-economic, demographic and health characteristics of the household. This is possible because the DHS surveys were carried out in standardized form, with the same list of socio-economic and demographic characteristics.

The DHS samples for the three countries were drawn through stratified clustered sampling comprising some 18000 children in 835 clusters. Although not available in the dataset, we were able to obtain the district location of each cluster and can therefore base our spatial analysis on the 156 districts in the three countries. ${ }^{2}$

Undernutrition among children is usually measured by determining the anthropometric status of the child with most research focusing on children below six years of age (e.g. WHO, 2006). Researchers typically distinguish between three types of undernutrition: wasting or insufficient weight for height indicating acute undernutrition; stunting or insufficient height for age indicating chronic undernutrition; and underweight or insufficient weight for age which could be a result of both stunting and wasting (e.g.

\footnotetext{
${ }^{2}$ We cannot use later DHS surveys undertaken in the three countries as they either did not take place in the same year or the district information was not available.
} 
UNICEF, 1998). Wasting, stunting, and underweight for a child $i$ are typically determined using a Z-score which is defined as:

$$
Z_{i}=\frac{A I_{i}-M A I}{\sigma}
$$

where AI refers to the individual anthropometric indicator (e.g. height at a certain age), MAI refers to the median of a reference population, and refers to the standard deviation of the reference population. The reference standard typically used for the calculation is the National Center for Health Statistics (NCHS)-Center for Disease Control (CDC) Growth Standard that has been recommended for international use by World Health Orgainasation (WHO, 1983; 1995). ${ }^{3}$

The percentage of children whose Z-scores were below -2 standard deviations (SD) from the median of the reference category are considered as undernourished (stunted, wasted, and underweight, depending on the indicator chosen), while those with Z-Scores below -3 are considered severely undernourished. In this paper we focus on stunting as our covariates were better able to explain chronic than acute undernutrition. We used the Z-Score (in a standardized form) as a continuous variable to use the maximum amount of information available in the data set.

\footnotetext{
${ }^{3}$ Recently, a new reference standard has been generated from which Z-scores can be calculated. For the purpose of this paper, the use of the new reference standard would not change the qualitative results. For a discussion of the new reference standard, see WHO (2006) and Klasen (2007).
} 


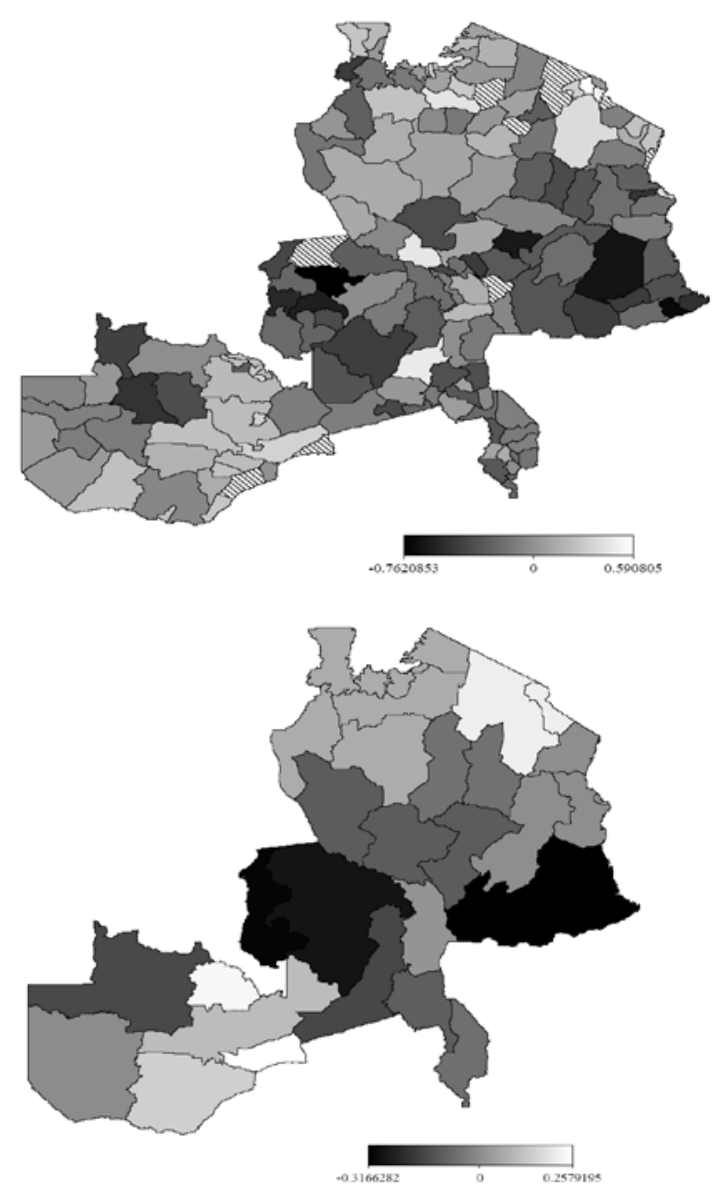

Figure 1 Observed mean Z-score of stunting by districts (left) and regions (right).

Note: Darker areas indicate low Z-Scores and thus poor undernutrition, those lighter ones suggest high ZScores. Moreover, hashed areas refer to districts for which no information was available in the data set.

The geographical distribution of the standardized Z-scores ${ }^{4}$ for the response variable stunting, averaged by district (left) and region (right), is displayed in Figure 1. It shows distinct spatial patterns of undernutrition. While in South-Western Zambia and Northern Tanzania, it appears that stunting is lower, there seem to be more areas of high stunting in North-Eastern Zambia, Northern Malawi and Southern Tanzania. In addition to local small-area variability, there might also be an underlying smooth spatial component which crosses borders, something we investigate below. The comparison between the left and right panel of Figure 1 also suggests that it is well worth examining the spatial pattern of

\footnotetext{
${ }^{4}$ The standardized Z-score calculated as the actual Z-score minus its median divided by its standard deviation was used for computation purposes.
} 
undernutrition at a more disaggregated district level as the regional analysis gloss over important intra-regional differentials.

Figure 2 shows a histogram and kernel density estimates of the distribution of the Z-scores, together with a normal density, with mean and variance estimated from the sample. This gives clear evidence that a Gaussian regression model is a reasonable choice for inference.
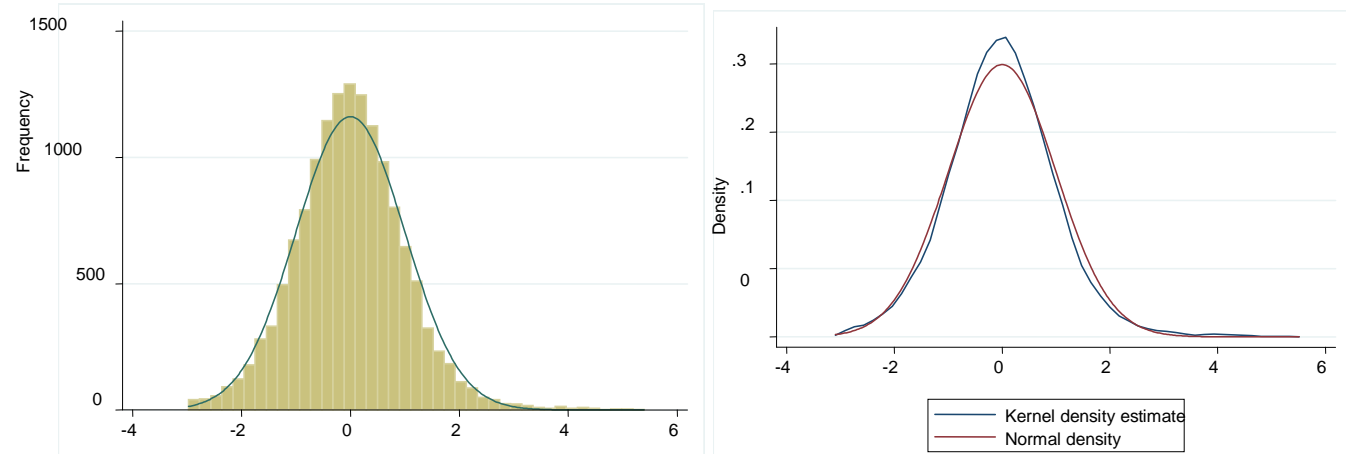

Figure 2 Histogram (left) and kernel density estimates (right) of "stunting”

Regarding the covariates, we were guided by the previous literature on the subject and the conceptual framework outlined in UNICEF (1998). Among the underlying determinants of chronic undernutrition, we consider socio-economic factors measured by an asset index, household size, the nutritional status of the mother (measured by her Body Mass Index), and access to electricity, health knowledge and care practices measured by mother's education, mother's marital status, birth interval, place of delivery, and vaccinations, and access to a radio. We also control for the sex of the child, urban-rural location, and the age of child. Based on prior own work as well as other literature (e.g. Kandala et al. 2001; 2002; WHO 1995; Moradi and Klasen, 2000), we investigate a potentially non-linear pattern of effects of the mother's BMI as well as the age pattern on 
undernutrition. For illustration, the empirical distribution of the stunting Z-score by child's age is shown in Figures 3. It is obvious that the effect of child's age on the mean Z-score of stunting is nonlinear. It will be difficult to model the possibly nonlinear effect of such covariates through a parametric functional form, which well justifies our use of a flexible semi-parametric model.

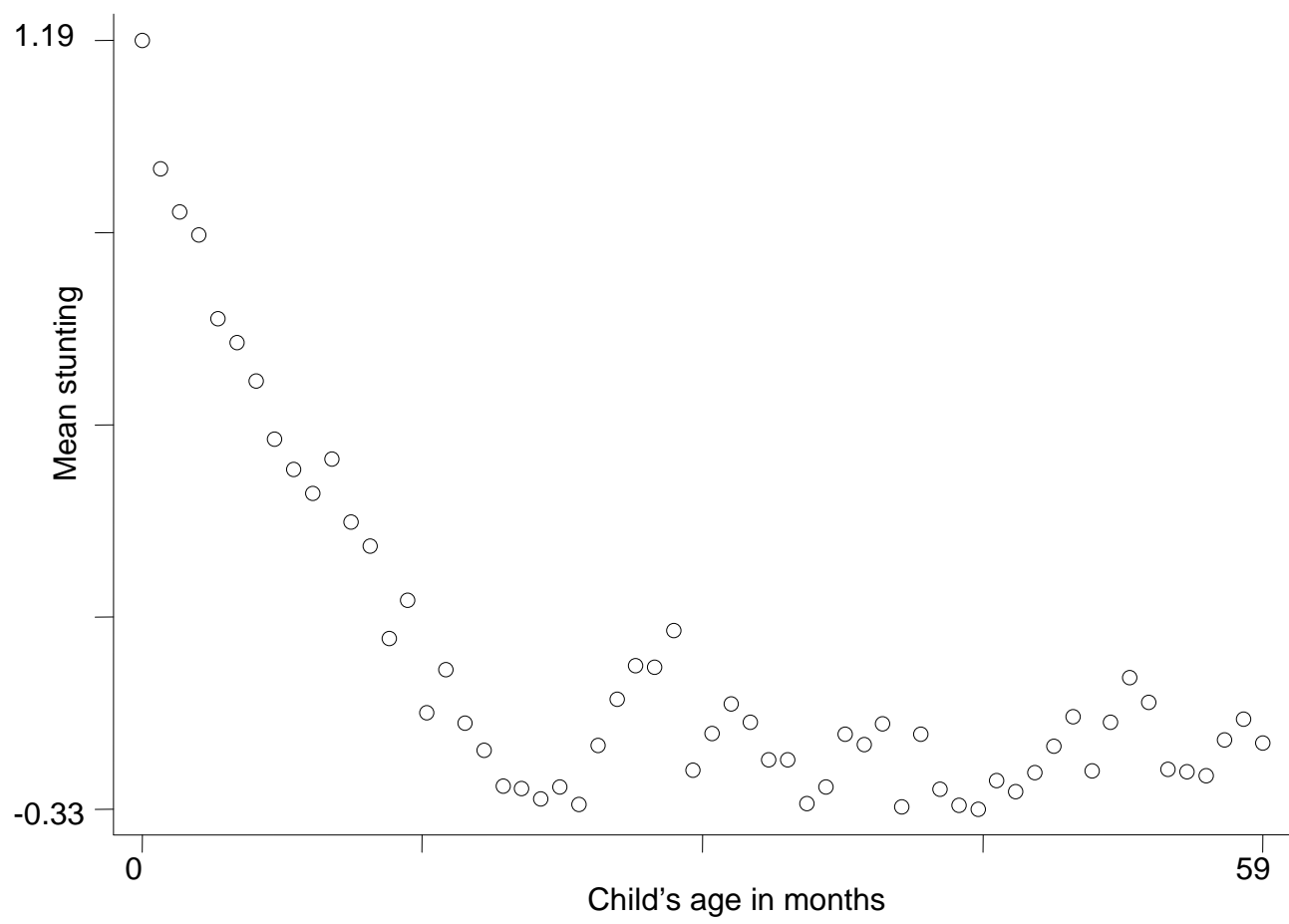

Empirical distributions of categorical covariates, together with coding used in the analysis, are given in Table 1. Most of the factors are placed in categories to be comparable to previous studies. For instance, household size is split into 'Small households of < than 6 members' (reference), 'Medium households of 6-10 members' and 'Large households of $>10$ members' in the three countries. Ownership of consumer items, such as a radio or car, as well as characteristics of the dwelling such as floor or roof type, toilet facilities and water source are items that measure poverty in these setting and the World Bank and others have used these items to generate an asset index, using Principal Components Analysis (PCA) (Filmer and Pritchett (2001). We use the first 
principal component derived from the pooled data from the three countries to obtain the index for each household. We sort children by the asset index and establish cut-off values for percentiles of the population. We then refer to the bottom third as 'low socioeconomic status', the next third as 'medium socioeconomic status', the top third as 'high socioeconomic status' (see Table 1). ${ }^{5}$

While all three countries do relatively poorly on the reported socioeconomic indicators, there are significant differences between the countries as well. In particular, households in Zambia appear to be better off in terms of access to electricity, radio, and female educational attainment. Income as proxied by the asset index and education levels are also higher in Zambia (Table 1). This country is also more heavily urbanized than the other two. Malawi and Tanzania are more similar, with Malawi doing somewhat worse on access to electricity. Malawi also has worse educational attainment at the lower levels but slightly higher among the highest levels than Tanzania. Since the effect of education and the asset index might vary across countries, it seems appropriate to test for interactions for these variables.

\footnotetext{
${ }^{5}$ Other categorical covariates, such as employment situation of the mother and type of toilet facility, turned out to be non-significant in the preliminary data analysis and are thus omitted.
} 
Table 1 Overview of Covariates

\begin{tabular}{|c|c|c|c|c|}
\hline Factor & Malawi (\%) & Tanzania(\%) & Zambia (\%) & Coding \\
\hline \multicolumn{5}{|l|}{ Residence } \\
\hline Rural & $74.5 \%$ & $84.3 \%$ & $57.3 \%$ & 0 : rural, reference \\
\hline Urban & $25.5 \%$ & $15.7 \%$ & $42.7 \%$ & 1: urban \\
\hline \multicolumn{5}{|l|}{ Has radio } \\
\hline No & $54.8 \%$ & $64.2 \%$ & $57.2 \%$ & 0: reference \\
\hline Yes & $45.0 \%$ & $34.1 \%$ & $42.4 \%$ & 1 \\
\hline \multicolumn{5}{|l|}{ Has electricity } \\
\hline No & $94.8 \%$ & $92.8 \%$ & $80.5 \%$ & 0 : reference \\
\hline Yes & $5.0 \%$ & $5.6 \%$ & $19.2 \%$ & 1 \\
\hline \multicolumn{5}{|l|}{ Educational attainment } \\
\hline No education & $41.6 \%$ & $37.2 \%$ & $17.9 \%$ & 0 : reference \\
\hline Incomplete primary & $42.8 \%$ & $18.9 \%$ & $30.3 \%$ & \\
\hline Complete primary & $10.1 \%$ & $40.6 \%$ & $32.8 \%$ & 1 (incl. inc. prim.) \\
\hline Incomplete secondary & $3.5 \%$ & $3.0 \%$ & $15.3 \%$ & \\
\hline Completed secondary & $1.7 \%$ & $0.1 \%$ & $2.1 \%$ & 2 (incl. inc. \& higher) \\
\hline Higher & $0.2 \%$ & $0.2 \%$ & $1.5 \%$ & \\
\hline \multicolumn{5}{|l|}{ Sex of child } \\
\hline Female & $49.3 \%$ & $49.9 \%$ & $49.9 \%$ & 0 : reference \\
\hline Male & $50.7 \%$ & $50.1 \%$ & $50.1 \%$ & 1: male \\
\hline \multicolumn{5}{|l|}{ Child's place of delivery } \\
\hline Born at home & $62.5 \%$ & $50.2 \%$ & $49.6 \%$ & 0 : reference \\
\hline Born in hospital & $37.5 \%$ & $49.8 \%$ & $60.4 \%$ & 1: hospital \\
\hline \multicolumn{5}{|l|}{ Current marital status } \\
\hline Single mothers & $11.5 \%$ & $15.3 \%$ & $15.2 \%$ & 1: single \\
\hline Married women & $88.5 \%$ & $84.7 \%$ & $84.8 \%$ & 0 : reference \\
\hline \multicolumn{5}{|l|}{ Child's receive vaccination } \\
\hline No & $22.1 \%$ & $24.1 \%$ & $27.18 \%$ & 0 : reference \\
\hline Yes & $77.5 \%$ & $75.9 \%$ & $72.8 \%$ & 1 \\
\hline \multicolumn{5}{|l|}{ Asset index } \\
\hline High socioeconomic status & $31.5 \%$ & $23.4 \%$ & $50.4 \%$ & 0 : reference \\
\hline Low socioeconomic status & $24.3 \%$ & $38.9 \%$ & $32.4 \%$ & 1: low \\
\hline Medium socioeconomic status & $44.2 \%$ & $37.8 \%$ & $17.2 \%$ & 2: medium \\
\hline \multicolumn{5}{|l|}{ Birth interval } \\
\hline Short: $\leq 24$ months & $23.85 \%$ & $21.34 \%$ & $22.84 \%$ & 0 : reference \\
\hline Long: $>24$ months & $76.15 \%$ & $78.66 \%$ & $77.16 \%$ & 1:long birth \\
\hline \multicolumn{5}{|l|}{ Size of household } \\
\hline Small size: $<6$ members & $46.72 \%$ & 32.40 & 33.45 & 0 : reference \\
\hline Medium size: 6-10 members & $47.23 \%$ & 50.37 & 53.94 & 1 \\
\hline Large size: > 10 members & $6.05 \%$ & 17.23 & 12.61 & 2 \\
\hline Mean of BMI & 21.96 & 21.75 & 21.96 & metrical \\
\hline District & 32 & 62 & 62 & spatial covariate \\
\hline
\end{tabular}

\section{BAYESIAN GEO-ADDITIVE REGRESSION MODELS}

Spatial analyses of undernutrition often are confined to using region-specific dummy variables to capture the spatial dimension. Here, we go a step further by exploring regional patterns of childhood undernutrition and, possibly nonlinear, effects of other factors within a simultaneous, coherent regression framework using a geo-additive semiparametric mixed model. Because the predictor contains usual linear terms, nonlinear effects of metrical covariates and geographic effects in additive form, such models are also called geo-additive models. Kammann and Wand (2003) proposed this type of models within an empirical Bayesian approach. Here, we apply a fully Bayesian approach 
as suggested in Fahrmeir and Lang (2001), Lang and Brezger (2004) which is based on Markov priors and uses MCMC techniques for inference and model checking.

Classical linear regression models of the form

$y_{i}=w_{i}{ }^{\prime} \gamma+\varepsilon_{i}, \quad \varepsilon_{i} \sim N\left(0, \sigma^{2}\right)$,

for observations $\left(y_{i}, w_{i}\right), i=1, \ldots, n$, on a response variable $y$ and a vector $w$ of covariates assume that the mean $E\left(y_{i} \mid w_{i}\right)$ can be modeled through a linear predictor $w_{i}^{\prime} \gamma$. In our application to childhood under-nutrition and in many other regression situations, we are facing the following problems: First, for the continuous covariates in the data set, the assumption of a strictly linear effect on the response $y$ may not be appropriate. In our study, such covariates are the child's age (age), the mother's age at birth (mab), and the mother's body mass index (BMI). Generally, it will be difficult to model the possibly nonlinear effect of such covariates through a parametric functional form, which has to be linear in the parameters, prior to any data analysis.

Second, in addition to usual covariates, geographical small-area information was given in form of a location variable $s$, indicating the region, district or community where individuals or units in the sample size live or come from. In our study, this geographical information is given by the districts of the three countries Malawi, Tanzania and Zambia. Attempts to include such small-area information using district-specific dummy-variables would in our case entail more than 200 dummy-variables and using this approach we would not assess spatial inter-dependence. The latter problem cannot also be resolved through conventional multilevel modeling using uncorrelated random effects (Goldstein, 1999). It is reasonable to assume that areas close to each other are more similar than areas far apart, so that spatially correlated random effects are required. 
To overcome these difficulties, we replace the strictly linear predictor through a geoadditive predictor, leading to the geo-additive regression model

$y_{i}=f_{1}\left(x_{i 1}\right)+\ldots+f_{p}\left(x_{i p}\right)+f_{s p a t}\left(s_{i}\right)+w^{\prime}{ }_{i} \gamma+\varepsilon_{\mathrm{i}} \quad$.

here, $f_{1}, \ldots, f_{p}$ are non-linear smooth effects of the metrical covariates, and $f_{\text {spat }}$ is the effect of the spatial covariate $s_{i} \in\{1, \ldots, S\}$ labelling the districts in the three countries. Regression models with predictors as in (2) are sometimes referred to as geo-additive models. The observation model (2) may be extended by including interaction $f(x) w$ between a continuous covariate $x$ and a binary component of $w$, say, leading to so called varying coefficient models, or by adding a nonlinear interaction $f_{1,2}\left(x_{1}, x_{2}\right)$ of two continuous covariates.

In a Bayesian approach unknown functions $f_{j}$ and parameters $\gamma$ as well as the variance parameter $\sigma^{2}$ are considered as random variables and have to be supplemented with appropriate prior assumptions. In the absence of any prior knowledge we assume independent diffuse priors $\gamma_{j} \propto$ const, $j=1, \ldots, r$ for the parameters of fixed effects. Another common choice is highly dispersed Gaussian priors.

Several alternatives are available as smoothness priors for the unknown functions $f_{j}\left(x_{j}\right)$, see Fahrmeir and Lang (2001), Fahrmeir, Kneib and Lang (2004). We use Bayesian $\mathrm{P}$ (enalized) - Splines, introduced by Eilers and Marx in a frequentist setting. It is assumed that an unknown smooth function $f_{j}\left(x_{j}\right)$ can be approximated by a polynomial spline of low degree. The usual choices are cubic splines, which are twice continuously differentiable piecewise cubic polynomials defined for a grid of $\mathrm{k}$ equally spaced knot $p$ on the relevant interval $[a, b]$ of the x-axis. Such a spline can be written in terms of a linear combination B-spline basis functions $B_{m}(x)$, i.e. 


$$
f(x)=\sum_{m=1}^{l} \beta_{m} B_{m}(x)
$$

These basis functions have finite support on four neighbouring intervals of the grid, and are zero elsewhere. A comparably small number of knots (usually between 10 and 40 ) is chosen to ensure enough flexibility in combination with a roughness penalty based on second order difference of adjacent B-spline coefficients to guarantee sufficient smoothness of the fitted curves. In our Bayesian approach this corresponds to second order random walks

$\beta_{m}=2 \beta_{m-1}-\beta_{m-2}+u_{m}$,

with Gaussian errors $u_{m} \sim N\left(0, \tau^{2}\right)$. The variance parameter $\tau^{2}$ controls the amount of smoothness, and is also estimated from the data. More details on Bayesian P-Splines can be found in Lang and Brezger (2004). Note that random walks are the special case of BSplines of degree zero.

We now turn our attention to the spatial effects $f_{\text {str }}$ and $f_{\text {unstr. }}$. For the spatially correlated effect $f_{\mathrm{str}}(\mathrm{s}), \mathrm{s}=1, \ldots \mathrm{S}$, we choose Markov random field priors common in spatial statistics (Besag, et al. 1991). These priors reflect spatial neighbourhood relationships. For geographical data one usually assumes that two sites or regions $\mathrm{s}$ and $\mathrm{r}$ are neighbours if they share a common boundary. Then a spatial extension of random walk models leads to the conditional, spatially autoregressive specification

$$
f_{s t r}(s) \mid f_{s t r}(r), r \neq s \sim N\left(\sum_{r \in \partial_{s}} f_{s t r}(r) / N_{s}, \tau^{2} / N_{s}\right)
$$

where $N_{s}$ is the number of adjacent regions, and $r \in \partial_{s}$ denotes that region $\mathrm{r}$ is a neighbour of region $s$. Thus the (conditional) mean of $f_{s t r}(s)$ is an average of function evaluations $f_{s t r}(s)$ of neighbouring regions. Again the variance $\tau_{\text {str }}^{2}$ controls the degree of smoothness. 
For a spatially uncorrelated (unstructured) effect $f_{\text {unstr }}$ a common assumption is that the parameters $f_{\text {unstr }}(\mathrm{s})$ are i.i.d. Gaussian

$f_{\text {unstr }}(\mathrm{s}) \mid \tau_{\text {unstr }}^{2} \sim N\left(0, \tau_{\text {unstr }}^{2}\right)$

Variance or smoothness parameters $\tau_{j}^{2}, j=1, \ldots, p$, str, unstr, are also considered as unknown and estimated simultaneously with corresponding unknown functions $f_{j}$. Therefore, hyper-priors are assigned to them in a second stage of the hierarchy by highly dispersed inverse gamma distributions $p\left(\tau_{j}^{2}\right) \sim \operatorname{IG}\left(a_{j}, b_{j}\right)$ with known hyper-parameters $a_{j}$ and $b_{j}$. For model choice, we routinely used the Deviance Information Criterion (DIC) developed in Spiegelhalter et. al. (2001) as a measure of fit and model complexity.

\section{RESULTS}

Based on previous analysis carried out separately for each country (Kandala et al., 2001), we choose a geo-additive model with interactions between country-effects and educational attainment as well as the asset index. Taking Tanzania as the reference country, we arrived at the model with interaction terms in Zambia and Malawi for the three levels of education and asset indices (see Table 1)

$y_{i}=f_{1}\left(a g c_{i}\right)+f_{2}\left(b m i_{i}\right)+f_{\text {str }}\left(s_{i}\right)+f_{\text {unstr }}\left(s_{i}\right)+w^{\prime}{ }_{i} \gamma+\varepsilon_{i}$

The model assumes that $f_{1}(), f_{2}()$ and $f_{\text {str }}$ are nonlinear effects and spatial effects were the same for all three countries. This was confirmed by prior separate analyses of the non-linear effects in each of the countries, which were found to be remarkably similar. 
Table 2 Posterior mean of fixed effects

\begin{tabular}{|c|c|c|c|}
\hline Variable & mean & $10 \%$ quant. & $90 \%$ quant. \\
\hline Constant & -0.14 & -0.22 & -0.07 \\
\hline Urban & 0.15 & 0.11 & 0.21 \\
\hline Male & -0.10 & -0.12 & -0.08 \\
\hline Incomplete primary \& complete primary education in Tanzania & 0.03 & -0.01 & 0.06 \\
\hline Incomplete \& complete secondary and higher in Tanzania & 0.35 & 0.24 & 0.46 \\
\hline Additive effect of incomplete \& complete primary education in Malawi & 0.08 & -0.04 & 0.12 \\
\hline Additive effect of incomplete \& complete secondary and higher in Malawi & 0.07 & -0.02 & 0.13 \\
\hline Additive effect of incomplete \& complete primary education in Zambia & -0.003 & -0.06 & 0.05 \\
\hline Additive effect of incomplete \& complete secondary and higher in Zambia & -0.17 & -0.30 & -0.05 \\
\hline Middle household in Tanzania & 0.01 & -0.02 & 0.05 \\
\hline Rich household in Tanzania & 0.18 & 0.14 & 0.23 \\
\hline Additive effect of middle household in Malawi & -0.05 & -0.11 & -0.01 \\
\hline Additive effect of rich household in Malawi & -0.06 & -0.13 & 0.02 \\
\hline Additive effect of middle household in Zambia & -0.01 & -0.07 & 0.06 \\
\hline Additive effect of rich household in Zambia & -0.02 & -0.09 & 0.05 \\
\hline Single mothers & -0.07 & -0.10 & -0.04 \\
\hline Long birth interval (>24 months) & 0.08 & 0.06 & 0.11 \\
\hline Medium household (6-10 members) & 0.03 & -0.004 & 0.05 \\
\hline Large household (>10 members) & 0.07 & 0.03 & 0.09 \\
\hline
\end{tabular}

Note: Left-out categories are rural, female, no education, poor households in Tanzania, married women, short birthinterval, and small households.

Table 2 contains the fixed effects, and the non-linear effects of BMI and child's age are shown in Figure 4. In the left-hand map of Figure 5 we show the mean Z-scores by district predicted by considering the socioeconomic covariates only; in the right-hand map we then subtract these predicted Z-score from the left-hand figure from the raw Zscore to generate the residuals by district that are not explained by the socioeconomic variables. These are then allocated to structured and unstructured effects. The posterior mean estimate of the structured spatial effects $f_{s t r}$ is shown in the maps of Figures 6 . Figure 7 shows the unstructured spatial effects. ${ }^{6}$ In addition, posterior probability maps indicate significance of the spatial effects (white/black = significantly positive/negative effect on the Z-score, grey = not significant). Note that the spatial effects are centred around zero, i.e. the average over all districts is zero, while the overall level is estimated through the intercept term. Before commenting on the substantive results, it is important to point out this model had the best fit after evaluation of the fit criteria using Deviance Information Criteria (DIC).

\footnotetext{
${ }^{6}$ The unstructured effects are smaller in magnitude and none are significant so that we do not include a significance map with this figure but briefly comment on them.
} 
The results for the fixed effects in Table 2 suggest that female children are slightly less stunted which had also been found in other studies (Klasen, 1996; Kandala et al., 2001; Kandala et al. 2006). This is indicated by the fact that the corresponding posterior mean, -0.09 for male, is negative and the $10 \%$ and $90 \%$ quantiles are both negative - indicating that the effect is statistically significant. It also is the case that children living with both parents (married women) are less stunted than other children, apparently benefiting from extra care of both parents. Alternatively couples may benefit from economies of scales for child care as well as in expenditures (Kandala, 2002; Kandala et al. 2006). Children from low socioeconomic households were, as expected, more stunted than children from high income backgrounds. The prior birth interval matters for the nutritional status of the child. The analysis shows that children born after a long birth interval are better off than other children.

Better nutritional status is observed in households with a large number of adult members. The impact of the household's size should, however, not be over-interpreted, since to some extent it directly mirrors infant mortality. For instance, a household with high mortality risk will remain small. In contrast, a household's size might also reflect its wealth, as a rich household will attract occupants (Kandala, 2002). Again, in a large household, a child might benefit from the help of several adults. Large households may benefit from scale economies in time for childcare as well as in expenditures. Alternatively, they may have become better at raising children through accumulated experience (Christiansen and Alderman, 2001).

The only country interactions that turned out to be significant and thus were retained in the model are the interactions with mother's education and asset index. Here we find that the positive effect of high mother's education is much smaller in Zambia 
than in Tanzania. This might be related to the fact that the economic crises of the early 1990s in Zambia also affected more educated groups, who were unable to shield their children from these adverse conditions. Similarly, the positive effect of middle and high income household is also much smaller in Malawi and Zambia. It thus appears that these two socioeconomic indicators have a much larger effect in Tanzania than elsewhere.
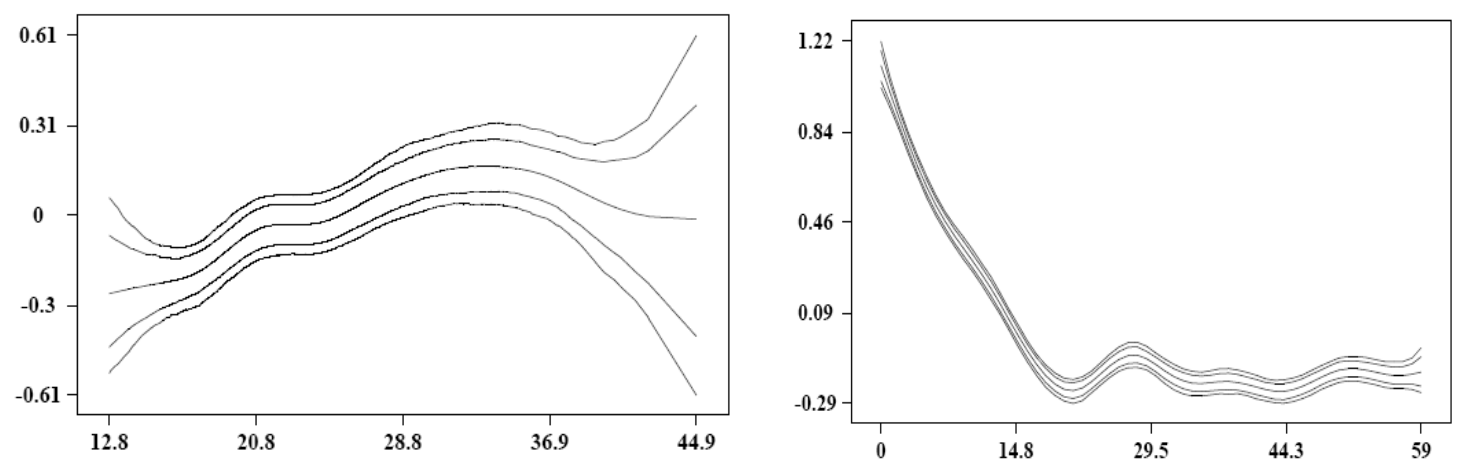

Figure 4 Non-linear effects of mother's body mass index (left) and child's age (right) on stunting

The left panel of Figure 4 shows the flexible modelling of the effect of the BMI of the mother. Shown are the posterior means together with $80 \%$ and 95\% pointwise credible intervals. We found the influence to be in the form of an inverse $\mathrm{U}$ shape. While the inverse $\mathrm{U}$ looks nearly symmetric, the descending portion exhibited a much larger range in the credible region. This appears quite reasonable as obesity of the mother (possibly due to a poor quality diet) is likely to pose less of a risk for the nutritional status of the child as very low BMIs which suggest acute undernutrition of the mother. The Zscore is highest (and thus stunting lowest) at a BMI of around 30-35.

The right panel of Figure 4 shows the effect of the child's age on its nutritional status. As suggested by the nutritional literature, we are able to discern the continuous worsening of the nutritional status up until about 20 months of age. This deterioration sets in right after birth and continues, more or less linearly, until 20 months. Such an 
immediate deterioration in nutritional status is not quite as expected as the literature typically suggests that the worsening is associated with weaning at around 4-6 months. One reason for this unexpected finding could be that, according to the surveys, most parents give their children liquids other than breast milk shortly after birth which might contribute to infections.

After 20 months the effect of age on stunting stabilizes at a low level. Through reduced growth and the waning impact of infections, children are apparently able to reach a low-level equilibrium that allows their nutritional status to stabilize. We also see a blip around 24 months of age. This is picking up the effect of a change in the data set that makes up the reference standard. Until 24 months, the currently used international reference standard is based on white children in the US of high socioeconomic status, while after 24 months; it is based on a representative sample of all US children (WHO, 1995). Since the latter sample exhibits worse nutritional status, comparing the Tanzanian children to that sample leads to a sudden improvement of their nutritional status at 24 months. This drawback of the reference standard is one reason for WHO's recent introduction of a new reference standard (WHO, 2006).

Figure 5 (left) shows that the socioeconomic effects are able to explain a fair amount of the spatial variation of undernutrition in the three countries. We calculate that the average residual spatial effect in the right-hand panel of Figure 5 is about 30\% lower than the original spatial effects plotted in Figure 1, showing that the socioeconomic effects explain some but not all of the spatial variation. 

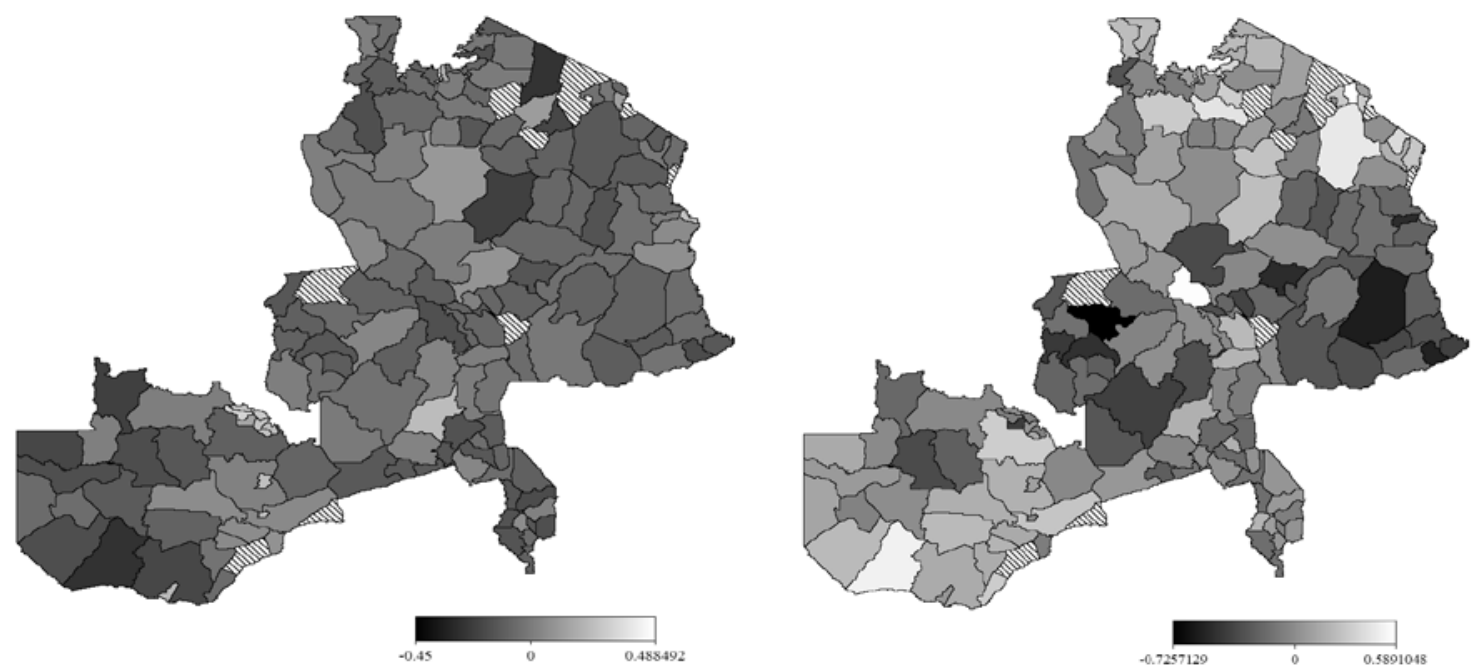

Light - low prevalence of stunting

Dark - high prevalence of stunting

Figure 5 Mean of stunting predicted by the covariates for Model 5 (left) and residual spatial effects of stunting (right).

However, the spatial residuals in the right-hand side of Figure 5 show that much of the variation in stunting remains to be explained. These spatial effects are then allocated by the model into structured effects which are shown in Figure 6 and unstructured residual effects in Figure 7.

Several important findings emerged. First, many of these structured spatial effects are significant as indicated by the probability map (Figure 6 right). The right panel of Figure 6 shows the posterior probability maps of undernutrition at a 95\% credible interval. The districts in black indicated a significant negative spatial effect (more undernutrition), while the districts in white imply a significant positive spatial effect. The rest of the districts (in grey) have no significant effect on undernutrition. Thus we clearly have a pattern of worse nutrition in Eastern and North-Eastern Zambia, Central Malawi, and Southern Tanzania. 
Conversely, Z-scores are significantly better in Northern Tanzania and South-Western Zambia. Second, while these structured effects suggest worse undernutrition in a belt ranging from Northern Zambia to Southern Tanzania, it is interesting to note that the districts in Northern Malawi are not significant components in that belt. Thus while some spatial residuals do spill significantly across borders, e.g. between Northern Zambia and Central Malawi, some borders do seem to matter in the sense that spatial residuals remain noticeably distinct in the analysis on the two sides of borders.

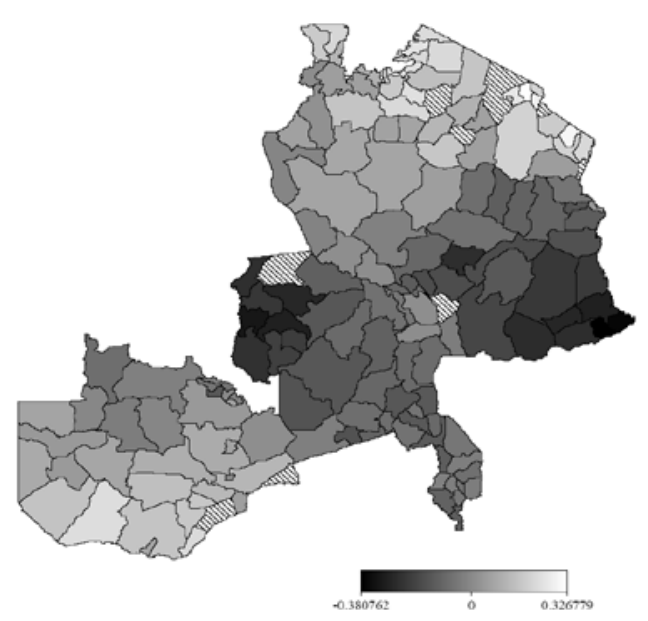

Light - low prevalence of stunting Dark - high prevalence of stunting

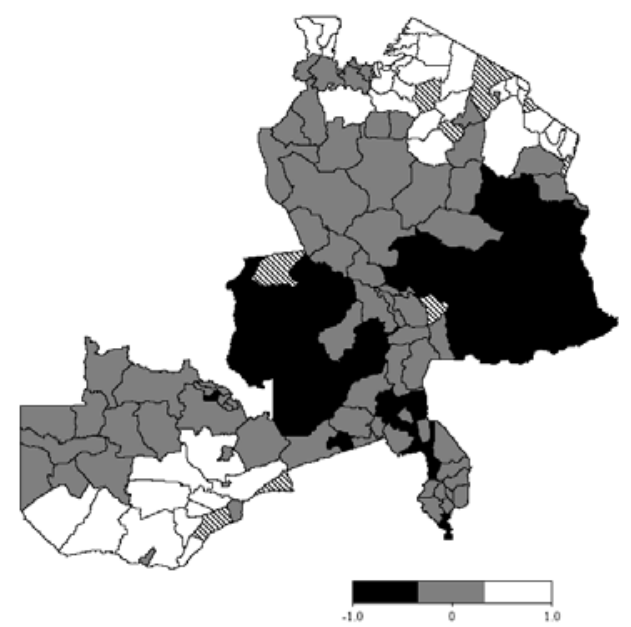

Black-significant negative effect Grey - not significant White-significant positive effect

Figure 6 Structured residual spatial effect of stunting (left) and posterior probabilities (right) of stunting for Model 5. 


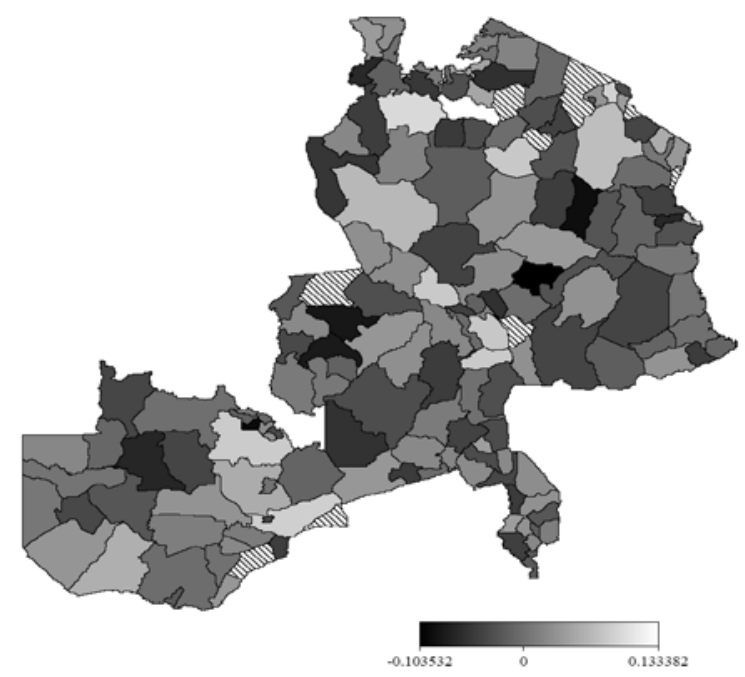

Figure 7 Unstructured spatial effects

Light - low prevalence of stunting

Dark - high prevalence of stunting

Third, the unstructured spatial effects shown in Figure 7, while being much smaller and not significant, also displayed an interesting pattern. While in Tanzania large cities have significantly higher Z-scores this was not the case in Zambia where some of the large cities in the Copperbelt (the small districts in the central North of the country) actually have lower Z-scores. This may be related to the effect of the decline in copper production and the impact of general economic decline and structural adjustment policies that have affected urban areas more than rural areas (World Bank, 2000).

The clear structured pattern begs for an explanation. None of the socioeconomic variables we tried in addition to the ones mentioned are able to reduce these pronounced spatial effects. One common factor to most of the areas that are negatively affected is that these areas are at comparatively low elevations. This distinction is most noticeable and clear in the South-North divide in Tanzania but it is also noticeable elsewhere. The difference could well be due to differences in disease prevalence such as Malaria, Schistosomasis, and other diseases that thrive at lower elevations and were particularly 
problematic along the Rift Valley. In an exploratory analysis, we compare the spatial pattern of prevalence of fever, diarrhoea; cough or any of the three illnesses combined with the structured spatial pattern and found that the spatial distribution of fever (presumably related to Malaria) has a fairly close resemblance to the structured spatial effects while the others did not appear to play a significant role. Future work should explore this linkage further. The measure of disease prevalence used here, recall of whether anyone in the household had been ill with fever, cough, or diarrhoea in the past 2 weeks is less than perfect as it is quite subjective, based on a short-term recall, and has considerable noise. Future work needs to address the question of disease environment more closely.

Moreover, the poor nutritional status in North-eastern Zambia could additionally be related to the poor access to health facilities and the general remoteness of these areas which are poorly served with transportation links (World Bank, 2000). These issues deserve closer attention and this procedure is merely able to highlight the important spatial patterns of undernutrition without being able to fully explain them.

Quite clearly, the methods used here are able to identify more subtle socioeconomic and spatial influences on undernutrition than reliance on linear models with regional dummy variables. As such, they are useful for diagnostic purposes to identify the need to find additional variables that can account for this spatial structure. Moreover, even if the causes of spatial structure are not fully explained, one can use this spatial information for poverty mapping and associated planning purposes, which is gaining increasing importance in policy circles that attempt to focus the allocation of public resources to the most deprived sections of the population. 


\section{CONCLUSIONS}

In this paper we pooled the 1992 Demographic and Health surveys of Malawi, Tanzania, and Zambia to model the socioeconomic and spatial determinants of undernutrition. We find strong support for our approach of flexibly modelling some covariates that clearly have non-linear influences and for including a spatial analysis. The spatial analysis shows distinct patterns that point to the influence of omitted variables with strong spatial structure or possibly epidemiological processes that account for this spatial structure.

The maps generated could be used for targeting development efforts or for exploring relationships between welfare indicators and others variables. For example, a mortality or undernutrition map could be overlaid with maps of other types of data, say on poverty, agro-climatic or other environmental characteristics. The visual nature of the maps may highlight unexpected relationships that would be overlooked in a standard regression analysis. These maps are novel tools to help policy-makers achieve Millennium Development Goals (MDGs) for child health in these countries.

\section{ACKNOWLEDGEMENTS:}

This research was supported by the Deutsche Forschungsgemeinschaft (German Research Foundation), and the Collaborative Research Center 386: Analysis of Discrete Structures. We thank participants at seminars in Munich, Tübingen, and at IFPRI in Washington DC, as well as two anonymous referees for helpful comments and discussions. We also want to thank Macro Int. for providing us with the district locations of the clusters in the three DHS data sets. 


\section{REFERENCES}

Besag, J., York, Y. and Mollie, A. (1991). Bayesian Image Restoration with two Applications in Spatial Statistics (with discussion). Ann. Inst. Statist. Math., 43, 1-59.

Borgoni, R. and Billardi, F.C. (2003). Bayesian spatial analysis of demographic survey data: An application to contraceptive use at first sexual intercourse. Demographic Research, 83, 61-92.

Caputo, A., Roraita,R., Klasen, S., and Pigeot, I. (2003) Undernutrition in Benin: An Analysis based on Graphical Models. Social Sciences and Medecine 56:1677-1691.

Christiaensen, L., \& Alderman, H. (2001). Child Malnutrition in Ethiopia: Can Maternal Knowledge Augment the role of Income?, World Bank, 2001.

Elbers, C., Lanjouw, J.O., Lanjouw, P. (2001). Welfare in Villages and Towns: MicroLevel Estimation of Poverty and Inequality. Tinbergen Institute Working paper No. 2000-0029/2, (at http://www.tinbergen.nl/.

Fahrmeir, L. and Lang, S. (2001). Bayesian Inference for Generalized Additive Mixed Models Based on Markov Random Field Priors. Applied Statistics (JRSS C), 50, 201220.

Filmer, D. and Pritchett, L.(2001) Estimating wealth effects without income of expenditure data-or tears: An application to Educational enrollment in states of India, Demography 38(1):115-132.

Goldstein, H. (1999). Multilevel statistical models. First Internet Edition. (http://multilevel.ioe.ac.uk/index.html.)

Guilkey, D. and Riphahn, R.(1998) The Determinants of Child Mortality in the Philippines: Estimation of a Structural Model. Journal of Development Economics 56 : 281-305.

Harttgen, K. and M. Misselhorn (2006). A multilevel approach to explain child mortality and undernutrition in South Asia and Sub-Saharan Africa. Mimeographed, University of Göttingen.

IMF (2000). A better world for all: progress towards the international development goals. International Monetary Fund, Organisation for Economic Co-operation and Development, United Nations, and World Bank, 2000.

Kammann EE, Wand MP. Geoadditive Models. Journal of the Royal Statistical Society C. 2003; 52: 1-18.

Kandala N-B (2002). Spatial modelling of Socio-Economic and Demographic Determinants of Childhood Undernutrition and Mortality in Africa, Ph.D Thesis, University of Munich, Shaker Verlag. 
Kandala N-B, S. Lang, S. Klasen, and L. Fahrmeir (2001). Semiparametric Analysis of the Socio-Demographic Determinants of Undernutrition in Two African Countries. Research in Official Statistics, EUROSTAT, Vol. 4 No.1:81-100.

Kandala N-B, M A Magadi and NJ Madise (2006): An Investigation of District Spatial Variations of Childhood Diarrhoea and Fever in Malawi. Social Science \& Medicine 62:1138-1152.

Klasen, S. (1996). Nutrition, Health, and Mortality in Sub-Saharan Africa: Is there a Gender Bias? Journal of Development Studies 32: 913-932.

Klasen, S. (1999). Malnourished and low mortality in South Asia, better nourished and dying young in Africa: What can explain this puzzle? SFB 386 Discussion Paper No. 214. University of Munich.

Klasen, S. Poverty, undernutrition, and child mortality: Some inter-regional puzzles and their implications for research and policy. Journal of Economic Inequality 2007 (published on-line in March, 2007; DOI 10.1007/s/10888-007-9056-x)

Lang S, Brezger A. Bayesian P-Splines. Journal of Computational and Graphical Statistics. 2004; 13: 183-212.

Moradi, A. and S. Klasen (2000). The Nutritional Status of Elites in India, Kenya, and Zambia: An Appropriate Guide for Developing International Reference Standards for Undernutrition? SFB 386 Discussion Paper No. 217. University of Munich.

Nyovani, J.M., Z. Matthews, and B. Margetts (1999). Heterogeneity of Child Nutritional Status between Households: A Comparison of six Sub-Saharan African Countries. Population Studies 53: 331-343.

Pelletier, D. (1998). Malnutrition, Morbidity, and Child Mortality in Developing Countries, In: United Nations (eds.) Too Young too Die: Genes or Gender? New York: United Nations.

Pritchett, L. and Summers, L. (1996). Wealthier is healthier. Journal of Human Resources, 31: 841-868.

Smith, L. and Haddad, L. (1999). Explaining Child Malnutrition in Developing Countries. IFPRI Research Report No. 111, Washington DC: IFPRI.

Smith, L. and Haddad, L. (2001). The Importance of Women's Status for Child Nutrition in Developing Countries. Mimeographed, IFPRI, Washington DC: IFPRI.

Stephenson, C. (1999). Burden of Infection on Growth Failure. Journal of Nutrition, Supplement, 534S-538S.

Spiegelhalter D., Best N., Carlin B., and Van der Line A. (2002). Bayesian measures of models complexity and fit. Journal of the Royal Stat. Soc. B(64):1-34. 
UNICEF (1998). The State of the World's Children. New York: UNICEF.

WHO (1983). Measuring Change in Nutritional Status. Geneva: WHO.

WHO (1995). Physical Status: The Use and Interpretation of Anthropometry. WHO Technical Report Series No. 854. Geneva: WHO.

World Bank (2000). Profile of poverty in Malawi, 1998. Poverty analysis of the Malawian Integrated Household Survey, 1997-98. Washington, DC: The World Bank.

World Health Organization (2006). Multicentre Growth Reference Study. Acta Paediatrica 450 (Suppl.), 1-87. 